\title{
SOME THEOREMS ON PERMUTATION POLYNOMIALS ${ }^{1}$
}

\author{
BY L. CARLITZ
}

Communicated by G. B. Huff, December 8, 1961

A polynomial $f(x)$ with coefficients in the finite field $G F(q)$ is called a permutation polynomial if the numbers $f(a)$, where $a \in G F(q)$ are a permutation of the $a$ 's. An equivalent statement is that the equation

$$
f(x)=a
$$

is solvable in $G F(q)$ for every $a$ in $G F(q)$. A number of classes of permutation polynomials have been given by Dickson [1]; see also Rédéi [3].

In the present note we construct some permutation polynomials that seem to be new. Let $q=2 m+1$ and put

$$
f(x)=x^{m+1}+a x .
$$

We define

$$
\psi(x)=x^{m},
$$

so that $\psi(x)=-1,+1$ or 0 according as $x$ is a nonzero square, a nonsquare or zero in $G F(q)$. Thus (2) may be written as

$$
f(x)=x(a+\psi(x)) .
$$

We shall show that for proper choice of $a$, the polynomial $f(x)$ is a permutation polynomial. We assume that $a^{2} \neq 1$; then $x=0$ is the only solution in the field of the equation $f(x)=0$. Now suppose (i) $f(x)$ $=f(y), \psi(x)=\psi(y)$. It follows at once from (4) that $x=y$. Next suppose (ii) $f(x)=f(y), \psi(x)=-\psi(y)$. Then (4) implies

$$
\psi\left(\frac{a+1}{a-1}\right)=-1
$$

If we take

$$
a=\frac{c^{2}+1}{c^{2}-1},
$$

where $c^{2} \neq \pm 1$ or 0 but otherwise is an arbitrary square of the field, it is evident that (5) is not satisfied. For $q \geqq 7$ such a choice of $c^{2}$ is

\footnotetext{
${ }^{1}$ Supported in part by National Science Foundation grant G-16485.
} 
possible. Hence $f(x)$ is a permutation polynomial for $q \geqq 7$ and $a$ defined by (6).

We show next that $f(x)$ is not a permutation polynomial for $G F\left(q^{r}\right)$, where $r>1$. For $r$ even this is evident since

$$
q^{2}-1 \equiv 0(\bmod m+1) .
$$

Replacing $r$ by $2 r+1$, put

$$
q^{2 r+1}=k(m+1)+m .
$$

Then expanding

$$
(f(x))^{k+m-1}=\left(x^{m+1}+a x\right)^{k+m-1}
$$

and reducing the result $\left(\bmod x^{2+1}-x\right)$, we find that the coefficient of $x^{q^{2 r+1}}-1$ is equal to

$$
\left(\begin{array}{c}
k+m-1 \\
m-1
\end{array}\right) a^{m-1} .
$$

Since by $(7) k \equiv 1(\bmod q)$, it follows that the binomial coefficient in (8) is congruent to $1(\bmod p)$. Therefore $f(x)$ is not a permutation polynomial for $G F\left(q^{2 r+1}\right)$.

We may state

Theorem 1. The polynomial

$$
f(x)=x^{m+1}+a x \quad(q=2 m+1)
$$

with a defined by (6) is a permutation polynomial for $G F(q)$ provided $q \geqq 7$. However it is not a permutation polynomial for any $G F\left(q^{r}\right)$ with $r>1$.

We consider next the case $q=3 m+1$ and again put $f(x)=x^{m+1}+a x$. It is now convenient to define

$$
\psi_{3}(x)=x^{m} .
$$

Thus for $x \in G F(q), x \neq 0$, we have $\psi_{3}(x)=1, \omega$ or $\omega^{2}$, where

$$
\omega^{2}+\omega+1=0 \quad(\omega \in G F(q)) .
$$

We assume first that $a \neq-1,-\omega,-\omega^{2}$. If we suppose (i) $f(y)=f(x)$, $\psi_{3}(x)=\psi_{3}(y)$, it follows that $x=y$. If we suppose (ii) $f(x)=f(y)$, $\psi_{3}(y)=\omega \cdot \psi_{3}(x)=\omega \lambda$, it follows that

$$
\psi_{3}\left(\frac{a+\lambda}{a+\omega \lambda}\right)=\omega .
$$

If we suppose (iii) $f(x)=f(y), \psi_{3}(y)=\omega^{2}, \psi_{3}(x)=\omega^{2} \lambda$ we get 


$$
\psi_{3}\left(\frac{a+\lambda}{a+\omega^{2} \lambda}\right)=\omega^{2} .
$$

Hence if we can choose $a$ so that

$$
\psi_{3}(a+1)=\psi_{3}(a+\omega)=\psi_{3}\left(a+\omega^{2}\right),
$$

both (10) and (11) will be contradicted.

Now (12) holds if and only if

$$
a+\omega=b^{3}(a+1), \quad a+\omega^{2}=c^{3}(a+1),
$$

where $b, c \in G F(q), b^{3} \neq 1, c^{3} \neq 1$. Eliminating $a$ we get

$$
b^{3}+\omega c^{3}+\omega^{2}=0 .
$$

Conversely if (14) is satisfied we get (13). By a theorem of Hurwitz which can be extended without difficulty to finite fields the number of solutions of (14) is asymptotic to $q$. This proves

TheOREM 2. For $q=3 m+1$ sufficiently large it is possible to choose $a \in G F(q)$ so that $f(x)=x^{m+1}+a x$ is a permutation polynomial for $G F(q)$.

It is not evident whether the second half of Theorem 1 can be carried over to this case.

Finally we state

THEOREM. Let $k$ be a fixed integer $\geqq 2$ and $q=k m+1$. Then there exists a constant $N_{k}$ and a number $a \in G F(q)$ such that

$$
f(x)=x^{m+1}+a x
$$

is a permutation polynomial for $G F(q)$ provided $q>N_{k}$.

The proof makes use of a theorem of Lang and Weil concerning the number of solutions of system of equations over a finite field [2].

\section{REFERENCES}

1. L. E. Dickson, Linear groups, New York, Dover, 1958.

2. S. Lang and A. Weil, Number of points of varieties in finite fields, Amer. J. Math. 76 (1953), 819-827.

3. L. Rédei, Über eindeutig umkehrbare Polynome in endlichen Körpern, Acta Sci. Math. Szeged. 11 (1946-1948), 85-92.

Duke University 